\title{
Recursos fisioterapêuticos nos desvios posturais da coluna vertebral: uma revisão integrativa
}

\author{
Physiotherapeutic resources in postural spinal \\ deviations: an integrative review
}

\author{
Maria Gerlane da Silva Freitas ${ }^{1}$ \\ Sara Maria Lopes de Medeiros ${ }^{2}$ \\ Gislainy Luciana Gomes Câmara ${ }^{3}$
}

${ }^{1}$ Autora para correspondência. Universidade Potiguar (Mossoró). Rio Grande do Norte, Brasil. algerlane24@gmail.com 2,3Universidade Potiguar (Mossoró). Rio Grande do Norte, Brasil. saramaria1313@gmail.com, gislainylgc@hotmail.com

\begin{abstract}
RESUMO | INTRODUÇÃO: Este estudo vai averiguar quais são as possibilidades de tratamento descritos na literatura para a escoliose, definida como curva lateral na coluna vertebral, apresentando uma leve rotação das vértebras. OBJETIVO: Identificar na literatura atual as principais técnicas fisioterapêuticas e seus benefícios para o tratamento da escoliose. MÉTODOS: Trata-se de uma revisão integrativa da literatura sobre os métodos fisioterapêuticos no tratamento da escoliose. Foram selecionados para esta revisão artigos publicados de 2009 a outubro de 2019 nas bases de dados eletrônicas Medline, Lilacs e SciELO, apenas artigos com tradução para o português foram considerados para esta revisão. RESULTADOS: Após a análise foram selecionados para esta revisão sete artigos para leitura na íntegra. Foram identificadas as seguintes condutas: técnica de pilates, reeducação postural global (RPG), isostretching, método klapp e cinesioterapia. Com os seguintes benefícios: melhora na postura, alinhamento da coluna vertebral torácica, melhora da flexibilidade, meIhora da assimetria do tronco, diminuição da intensidade da dor, redução da curva escoliótica, melhora postural no alinhamento de cabeça, ombros, escápulas, ângulo de Talles, tronco, abdômen e pelve, aumento da força dos músculos flexores e extensores de tronco e redução do ângulo de Cobb da escoliose. CONCLUSÃO: Existem diversos métodos da fisioterapia com benéficos para o tratamento da escoliose, destacando o RPG e o pilates para a melhora da força, flexibilidade, dor e redução da curva escoliótica.
\end{abstract}

PALAVRAS-CHAVE: Escoliose. Fisioterapia. Tratamento.

\begin{abstract}
INTRODUCTION: This study will evaluate what are the treatment possibilities described in the literature for a school, defined as a lateral curve in the spine, presenting a slight alteration of the vertebrae. OBJECTIVE: To identify in the current literature the main physicaltherapy techniques and their benefits for the treatment of scoliosis. METHODS: This is an integrative review of the literature on physicaltherapy methods in the treatment of scoliosis. Articles published in 2009 to October 2019 in the electronic databases Medline, Lilacs and SciELO were selected for this review, only articles with Portuguese translation that were submitted to this review. RESULTS: After the analysis, seven articles for reading in full were selected for this review. The following procedures were identified: pilates technique, global postural reeducation (RPG), isostretching, klapp method and kinesiotherapy. With the following benefits: improves posture, alignment of the vertebral thoracic spine, improves flexibility, improves asymmetry of the trunk, decreases the intensity of pain, reduces the educational curve, improves postural posture, head alignment, pain, scapulae and highs in height, trunk, abdomen and pelvis, increased strength of trunk flexor and extensor muscles and reduced Cobb angle of scoliosis. CONCLUSION: Several physiotherapy methods with benefits for the treatment of scoliosis, highlighting RPG and pilates for improvements in strength, flexibility, pain and reduction of school curves.
\end{abstract}

KEYWORDS: Scoliosis. Physiotherapy. Treatment. 


\section{Introdução}

Este estudo vai averiguar quais são as possibilidades de tratamento fisioterapêutico descritas na literatura sobre escoliose, esclarecer a importância e os benefícios da fisioterapia em pacientes com esta condição, reforçando as evidências científicas.

A coluna vertebral é uma importante estrutura para sustentação e manutenção da postura ereta, além da função de proteção da medula espinhal. É formada por um conjunto de 33 vértebras dividido por cinco regiões, cervicais, torácicas, lombares, sacro e cóccix. Nas curvaturas anatômicas de suas vértebras apresentam 2 cifoses (torácica e sacral) e 2 lordoses (cervical e lombar). Em sua constituição encontram-se inseridos diversos músculos e ligamentos que são importantes para a sua sustentação e funcionalidade ${ }^{1}$.

A coluna pode sofrer algumas alterações posturais, são elas: no plano anatômico sagital, como a hiperlordose lombar e cervical; em que ocorre acentuação da concavidade posterior das curvaturas, hipercifose torácica; quando há aumento da concavidade anterior da curvatura torácica, cifose lombar; diminuição da sua curvatura lordótica causando achatamento dessa região, e no plano frontal a escoliose; é caracterizada pelo desvio lateral da coluna vertebral podendo apresentar-se em forma de "C" curva única ou "S" curva dupla1,2.

A escoliose foi mencionada pela primeira vez por Hipócrates, cujo termo é derivado do grego e significa curvatura. É caracterizada por uma curvatura anormal da coluna vertebral, superior a $10^{\circ}$ segundo o método de $\mathrm{COBB}$, associada ou não à rotação dos corpos vertebrais nos planos sagital e axial. O ângulo de COBB mede a curvatura da coluna no plano coronal e as escolioses podem ser classificadas em leve (entre $10^{\circ} \mathrm{a}$ $20^{\circ}$ ), moderada (entre $20^{\circ}$ a $40^{\circ}$ ) e grave $\left(>50^{\circ}\right)^{2,3}$.
Divide-se em dois grupos: Funcional ou não estrutural e estrutural ou morfológica. $\mathrm{Na}$ escoliose funcional a curva é flexível e pode ser corrigida se o paciente se inclinar para o lado convexo. Geralmente não progridem e não apresentam rotação vertebral. As escolioses estruturais apresentam rotação e alterações anatômicas das vértebras, podendo progredir durante o crescimento. Na curvatura estrutural surge uma elevação devido a rotação das vértebras, chamado gibosidade, vista no lado da convexidade da curva e observado através do Teste de Adams, utilizado mundialmente para detecção da escoliose ${ }^{2,3}$.

A escoliose é classificada em três tipos: A congênita; quando o indivíduo nasce com ela, é decorrente de um desenvolvimento anormal das vértebras do feto. A neuromuscular; é causada por doenças, como paralisia cerebral e distrofia muscular. A idiopática; não possui causa conhecida, desenvolve-se durante a fase da puberdade ${ }^{3,4}$. Segundo a Sociedade de pesquisa em escoliose Scoliosis Research Society (SRS), a idiopática é responsável por $80 \%$ dos casos de escoliose, sendo as meninas adolescentes as mais afetadas. Em 30\% dos casos em adolescentes possuem histórico familiar de escoliose. Pode surgir entre $4 \mathrm{e}$ 10 anos de idade e representa $10 \%$ a $15 \%$ de toda escoliose idiopática juvenil ${ }^{5}$.

A Fisioterapia tem como principal objetivo realizar um tratamento conservador. É recomendada como primeira opção de tratamento para pequenas curvaturas da coluna, com a finalidade de impedir sua progressão. Também atuando no pré e pós-operatório de correção da coluna. Possui amplos recursos para o tratamento como, o método Klapp, cinesioterapia, estimulação elétrica dos músculos, órteses, osteopatia, reeducação postural global (RPG), pilates e Iso-stretching ${ }^{2,6}$. O objetivo do presente trabalho foi identificar na literatura atual as principais técnicas fisioterapêuticas e seus benefícios para o tratamento da escoliose. 


\section{Materiais e métodos}

\section{Estratégias de busca}

A busca foi realizada nas bases de dados eletrônicos Medline (National Library of Medicine), Lilacs (Literatura Latino-americana e do Caribe em Ciências da Saúde), e SCIELO (Scientific Electronic Library Online), entre os dias 10 de setembro de 2019 ao dia 05 de outubro de 2019. Foram utilizados os seguintes descritores: Escoliose, Fisioterapia e Tratamento, contidos nas bases Ciências da Saúde (DeCS) e Medical Subject Headings (MeSH) no idioma português, utilizando os operadores booleanos "AND" (E) e "OR" (OU). Foi utilizado como limite na estratégia de busca os estudos publicados entre os anos de 2009 até outubro de 2019, as palavras-chave pesquisadas constarem no título ou resumo e estarem disponíveis eletronicamente.

\section{Seleção dos estudos}

Para inclusão desta revisão, os estudos rastreados precisam estar de acordo com as seguintes razões: tratar de pesquisas com algum recurso fisioterapêutico aplicado em indivíduos com escoliose, está disponível nas bases científicas eletrônicas já descritas, terem sido publicados entre os anos de 2009 até outubro de 2019 e que possuam tradução para o idioma português. Para exclusão desta revisão, os estudos que não se adequarem ao tema proposto ou tratar de revisões da literatura serão excluídos da pesquisa.

\section{Análise dos artigos}

A busca foi realizada por dois revisores, que avaliaram os títulos e resumos independentemente; se existisse alguma discordância entre estes quanto à seleção dos artigos, esta seria resolvida por um terceiro revisor. Após esta primeira seleção, os artigos foram lidos na íntegra e aqueles que não estivessem dentro dos critérios estabelecidos foram excluídos do estudo.

\section{Descrição dos artigos}

Para organizar as informações dos artigos, foi realizada uma síntese das principais informações de cada estudo em um quadro que contém os seguintes tópicos: autor, característica da amostra, tipo de intervenção, tempo e duração da intervenção e resultados.

\section{Avaliação de qualidade}

Para realização da qualidade metodológica dos estudos incluídos nesta revisão, foi utilizado a escala de avaliação PEDro ${ }^{20}$. Os resultados estão descritos no quadro 3.

\section{Resultados}

Na pesquisa realizada nas bases de dados eletrônicas, foi seguida conforme a metodologia para revisões sistemáticas e meta-análises (PRISMA) demonstrada na (Figura 1) ${ }^{21}$. Nas bases do LILACS e SciELO foram identificados, respectivamente, em cada uma delas 27 e 26 artigos potencialmente relevantes. No MEDLINE não foi encontrado artigos em português. Após análise do título e dos resumos, foram excluídos, 24 do LILACS, 22 no SciELO, por não abordar o tema escolhido ou por serem artigos de revisão. Assim, conforme os critérios de inclusão e exclusão predeterminados, foram selecionados para o quadro sete artigos para leitura na íntegra, sendo, três do LILACS e quatro do SciELO. As características dos artigos selecionados estão descritas no quadro 1 e 2 . 
Figura 1. Fluxograma dos estudos selecionados nas bases de dados eletrônicas de acordo com a recomendação PRISMA

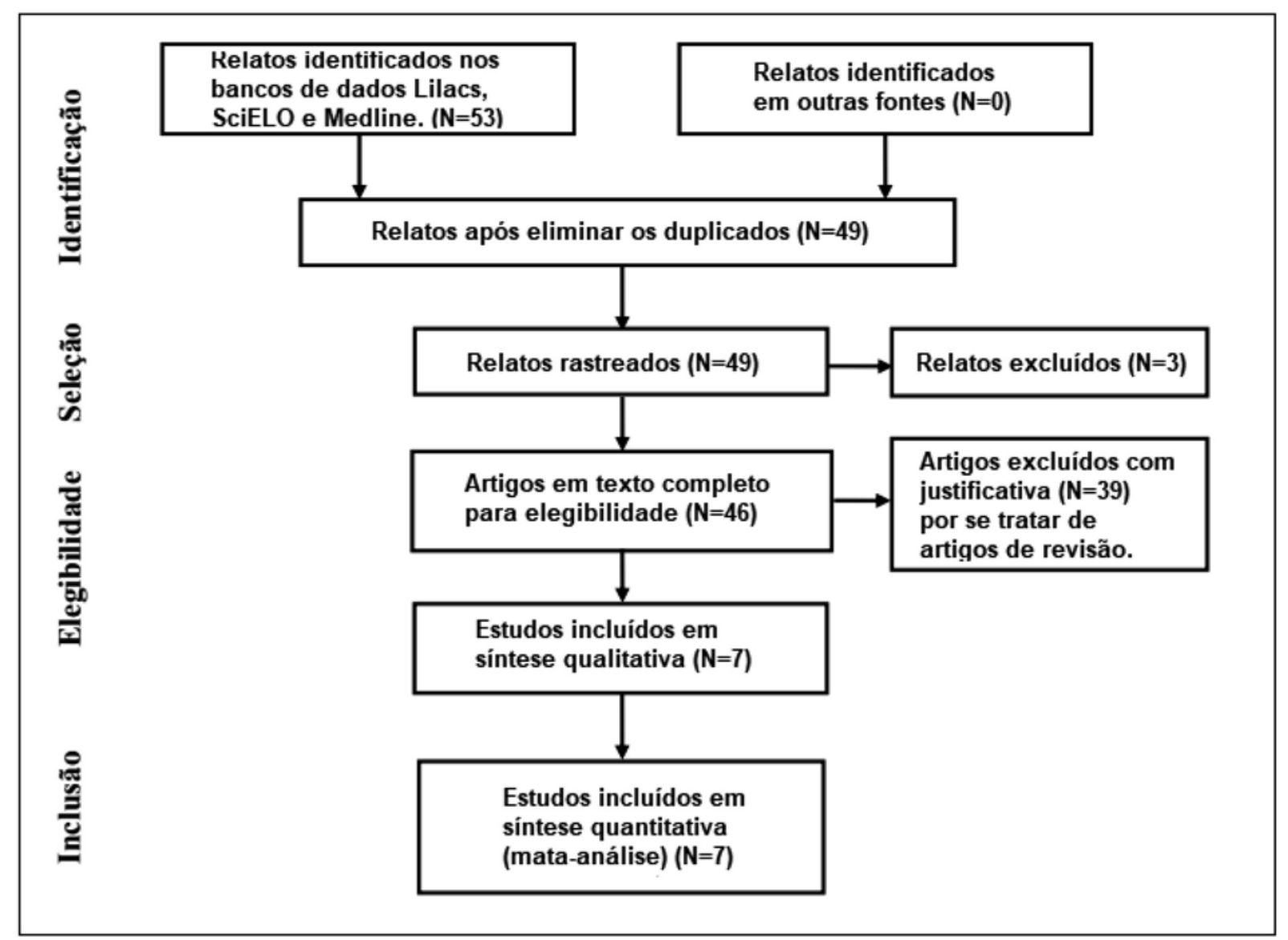




\begin{tabular}{|c|c|c|c|c|}
\hline Autor & $\begin{array}{c}\text { Característica da } \\
\text { amostra }\end{array}$ & Tipo de Intervenção & $\begin{array}{c}\text { Tempo e duração } \\
\text { da intervenção }\end{array}$ & Resultado \\
\hline Monte-Raso et $\mathrm{al}^{7}$ & $\begin{array}{l}12 \text { indivíduos com } \\
\text { idade média de } 20 \\
\text { anos e sexo não } \\
\text { revelado dividido em } \\
2 \text { grupos ( } 1 \text { com n=8: } \\
\text { com mais de } 30 \\
\text { atendimentos } \\
\text { em grupo } 2 \text { com n } \\
=4 \text { : com menos de } \\
30 \text { atendimentos) }\end{array}$ & Isostretching & $\begin{array}{l}\text { Frequência de } 3 \times \text { na } \\
\text { semana. Durante } 54 \\
\text { sessões com tempo } \\
\text { de } 60 \text { minutos }\end{array}$ & $\begin{array}{l}\text { Foi eficaz no } \\
\text { alinhamento da } \\
\text { coluna vertebral } \\
\text { torácica nos dois } \\
\text { grupos, bem como } \\
\text { na melhora da } \\
\text { flexibilidade no } \\
\text { grupo 1. Não foi } \\
\text { eficaz para } \\
\text { assimetrias posturais } \\
\text { no plano frontal } \\
\text { anterior e posterior. }\end{array}$ \\
\hline Lunes et $\mathrm{al}^{8}$ & $\begin{array}{l}16 \text { indivíduos com } \\
\text { idade média de } 15 \\
\text { anos com } 3 \\
\text { pacientes do sexo } \\
\text { masculino e } 13 \\
\text { pacientes do sexo } \\
\text { feminino }\end{array}$ & Método Klapp & $\begin{array}{l}\text { Duração de } 10 \\
\text { semanas com } \\
\text { frequência de } 2 \text { x na } \\
\text { semana. Durante } 20 \\
\text { sessões com tempo } \\
\text { de } 70 \text { minutos }\end{array}$ & $\begin{array}{l}\text { Apontam para a } \\
\text { melhora da } \\
\text { assimetria do tronco } \\
\text { e flexibilidade. Não } \\
\text { foi eficaz para } \\
\text { assimetrias da pelve, } \\
\text { alinhamento da } \\
\text { cabeça e não houve } \\
\text { modificações das } \\
\text { curvaturas vertebrais }\end{array}$ \\
\hline Toledo et al $^{9}$ & $\begin{array}{l}20 \text { indivíduos com } \\
\text { idade média de } 10 \\
\text { anos, } 11 \text { do sexo } \\
\text { masculino e } 9 \text { do } \\
\text { sexo feminino } \\
\text { dividido em } 2 \text { grupos } \\
\text { (RPG: com } n=10 \text { e GC } \\
\text { com } n=10 \text { ) }\end{array}$ & RPG & $\begin{array}{l}\text { Duração de } 12 \\
\text { semanas com } \\
\text { frequência de } 2 x \text { na } \\
\text { semana. Sessões } \\
\text { com tempo de } 25 \text { a } \\
30 \text { minutos }\end{array}$ & $\begin{array}{l}\text { O GRPG apresentou } \\
\text { diminuição } \\
\text { significativa no } \\
\text { ângulo da escoliose, } \\
\text { enquanto o GC } \\
\text { registrou aumento } \\
\text { não significativo }\end{array}$ \\
\hline Segura et al ${ }^{10}$ & $\begin{array}{l}8 \text { indivíduos com } \\
\text { idade média entre } \\
10 \text { e } 16 \text { anos do sexo } \\
\text { feminino }\end{array}$ & RPG & $\begin{array}{l}\text { Frequência de } 2 \times \text { na } \\
\text { semana. Durante } 40 \\
\text { sessões com tempo } \\
\text { de } 45 \text { minutos }\end{array}$ & $\begin{array}{l}\text { Redução significativa } \\
\text { do ângulo da } \\
\text { escoliose, mesmo não } \\
\text { registrando uma } \\
\text { diferença na } \\
\text { discrepância dos } \\
\text { membros inferiores }\end{array}$ \\
\hline Araújo et al ${ }^{11}$ & $\begin{array}{l}31 \text { indivíduos com } \\
\text { idade média entre } \\
18 \text { e } 25 \text { anos do sexo } \\
\text { feminino. Dividido } \\
\text { em } 2 \text { grupos } \\
\text { (Controle: } n=11 \text { e } \\
\text { Grupo Experimental: } \\
n=20 \text { ) }\end{array}$ & Pilates & $\begin{array}{l}\text { Duração de } 3 \text { meses } \\
\text { durante } 24 \text { sessões } \\
\text { com tempo de } 60 \\
\text { minutos }\end{array}$ & $\begin{array}{l}\text { Diminuição } \\
\text { significativa da } \\
\text { intensidade da dor no } \\
\text { grupo experimental }\end{array}$ \\
\hline $\begin{array}{l}\text { Miotti de Moura et } \\
\mathrm{al}^{12}\end{array}$ & $\begin{array}{l}1 \text { indivíduo com } \\
\text { idade média de } 11 \\
\text { anos do sexo } \\
\text { feminino }\end{array}$ & Pilates & $\begin{array}{l}\text { Duração de } 4 \\
\text { semanas com } \\
\text { frequência de } 3 \times \text { na } \\
\text { semana. Durante } 10 \\
\text { sessões com tempo } \\
\text { de } 60 \text { minutos }\end{array}$ & $\begin{array}{l}\text { Melhora postural no } \\
\text { alinhamento de } \\
\text { cabeça, ombros, } \\
\text { escápulas, ângulo de } \\
\text { Talles, tronco, } \\
\text { abdômen e pelve. } \\
\text { Aumento da } \\
\text { flexibilidade e a força } \\
\text { dos músculos } \\
\text { flexores e extensores } \\
\text { de tronco }\end{array}$ \\
\hline Fiorelli et $\mathrm{al}^{13}$ & $\begin{array}{l}1 \text { indivíduo com } \\
\text { idade média de } 11 \\
\text { anos do sexo } \\
\text { feminino }\end{array}$ & Cinesioterapia & Não revelado & $\begin{array}{l}\text { Redução acentuada } \\
\text { do ângulo da } \\
\text { escoliose }\end{array}$ \\
\hline
\end{tabular}

Fonte: dados da pesquisa (2019) 
O total de participantes incluídos nos 7 estudos abordados foram de 111 indivíduos, destes, 63 são do sexo feminino (56.76\%) e 48 do sexo masculino $(43,24 \%)^{8,9,10,11,12,13}$, sendo que um dos estudos incluídos não forneceram esta informação com 12 participantes de sexo não revelado7. Em dois estudos a amostra apresenta indivíduos com idade média entre 18 a 25 anos $^{7,11}$. Nos outros cinco estudos, os indivíduos apresentam idade igual ou menor que 15 anos $^{8,9,10,12,13}$, sendo que em dois destes estudos os participantes apresentam idade igual a 11 anos ${ }^{12,13}$.

Cada estudo teve uma duração diferente, um não revelou o tempo das intervenções ${ }^{13}$. Dois realizaram as intervenções com média de 10 a 12 sessões totalizando o tempo mínimo9,12. Dois realizaram com tempo médio de 20 a 24 sessões ${ }^{8,11}$, um realizou com 40 sessões $^{10}$ e um apresentou o tempo máximo de 54 sessões?

Os autores dos presentes estudos optaram pelo uso do ângulo de Cobb9,10,11,13 e outros por uma avaliação postural para o acompanhamento terapêutico ${ }^{7,8,12}$. Quatro destes apresentaram resultados numéricos em graus quanto ao ângulo de $\operatorname{Cobb}^{9,10,13}$ e um destes não apresentou esta informação nos resultados ${ }^{11}$, um estudo avaliou e apresentou a discrepância de membros inferiore ${ }^{10}$, outro a dor crônica na coluna ${ }^{11}$, três artigos avaliaram e apresentaram a avaliação postural fotografadas com marcação de pontos anatômicos ${ }^{7,8,12}$, dois destes avaliaram a flexibilidade ${ }^{7,12}$ e um a força dos músculos extensores e flexores de tronco ${ }^{12}$.

Quadro 2. Descrição das técnicas empregadas nos estudos selecionados

\begin{tabular}{|c|c|}
\hline Técnica / Autor & Descrição \\
\hline $\begin{array}{l}\text { Isostretching } \\
\text { Monte-Raso et } \mathrm{al}^{7}\end{array}$ & $\begin{array}{l}\text { Exercícios isométricos excêntricos, em que o tempo de manutenção das posturas é } \\
\text { regido por três expirações profundas e prolongadas, nas posições deitado, sentado, em } \\
\text { pé, utilizando bola e bastão. Um total de } 67 \text { posturas. }\end{array}$ \\
\hline $\begin{array}{l}\text { Método Klapp } \\
\text { lunes et } \mathrm{al}^{8}\end{array}$ & $\begin{array}{l}\text { Exercícios: relaxamento, engatinhar perto do chão, deslizamento horizontal, } \\
\text { deslizamento lateral, engatinhar lateral, arco grande, virar o braço e grande curva. }\end{array}$ \\
\hline $\begin{array}{l}\text { RPG } \\
\text { Toledo et al }{ }^{9}\end{array}$ & $\begin{array}{l}\text { Foram utilizadas as posturas "rã no chão" e "rã no ar". A evolução das posturas vai de } \\
\text { acordo com o controle da respiração, a capacidade de manter o alinhamento e } \\
\text { correções necessárias, dependendo basicamente das condições apresentadas por cada } \\
\text { indivíduo. }\end{array}$ \\
\hline $\begin{array}{l}\text { RPG } \\
\text { Segura et al }{ }^{10}\end{array}$ & Posturas sentada e rã no ar. \\
\hline $\begin{array}{l}\text { Pilates } \\
\text { Araújo et al }{ }^{11}\end{array}$ & $\begin{array}{l}\text { Aquecimento: caminhada de } 8 \text { minutos. Alongamento da coluna para frente, rolamento } \\
\text { por cima, puxar a perna de frente, prece maometana. Parte específica: foram utilizados } \\
\text { exercícios com bola Suíça e equipamentos próprios da técnica de pilates. Foram } \\
\text { realizados doze exercícios com } 10 \text { repetições cada. }\end{array}$ \\
\hline $\begin{array}{l}\text { Pilates } \\
\text { Miotti de Moura et al }{ }^{12}\end{array}$ & $\begin{array}{l}\text { Preparação: Rolamento para baixo em pé, alongamento em decúbito dorsal e decúbito } \\
\text { ventral. Parte específica: Foram utilizados exercícios específicos do método pilates solo e } \\
\text { com acessórios, } 10 \text { repetições para cada exercício. Volta à calma: Esse procedimento foi } \\
\text { composto de três movimentos, tendo duração de cinco minutos cada um. }\end{array}$ \\
\hline $\begin{array}{l}\text { Cinesioterapia } \\
\text { Fiorelli et } \mathrm{al}^{13}\end{array}$ & $\begin{array}{l}\text { Retroversão da pelve, } 10 \text { minutos. Pés sobre a bola Suiça e realização de elevação da } \\
\text { pelve } 12 \text { séries } 3 \text { vezes. Quadril em flexão de } 90^{\circ} \text {, joelhos levemente flexionados, } \\
\text { membros superiores flexionados e as mãos e os pés segurando a bola suíça com } 10 \\
\text { séries. Exercícios da série de Willians com } 8 \text { séries, mantendo por } 15 \text { segundos. }\end{array}$ \\
\hline
\end{tabular}

Fonte: dados da pesquisa (2019) 
De acordo com os artigos selecionados para esta revisão, foram identificados os seguintes benefícios após a realização dos exercícios de cada técnica proposta: melhora na postura, o alinhamento da coluna vertebral torácica ${ }^{7}$, melhora da flexibilidade ${ }^{7,8,12}$ melhora da assimetria do tronco ${ }^{8}$, diminuição da intensidade da dor ${ }^{11}$, melhora postural no alinhamento de cabeça, ombros, escápulas, ângulo de Talles, tronco, abdômen e pelve ${ }^{12}$, aumento da força dos músculos flexores e extensores de tronco ${ }^{12}$ e redução do ângulo da escoliose $\mathrm{e}^{9,10,13}$. Em alguns estudos ocorreu a não eficácia para assimetrias da pelve, alinhamento da cabeça e não houve modificações das curvaturas vertebrais, não eficaz para assimetrias posturais e não registrou diferença na discrepância dos membros inferiores ${ }^{7,8,10}$.

Quadro 3. Avaliação metodológica dos estudos através da escala de PEDro

\begin{tabular}{|c|c|c|c|c|c|c|c|c|c|c|c|}
\hline & 2 & 3 & 4 & 5 & 6 & 7 & 8 & 9 & 10 & 11 & TOTAL \\
\hline Monte-Raso et $\mathrm{al}^{7}$ & $x$ & & & $x$ & & & $x$ & $x$ & $x$ & $x$ & $6 / 10$ \\
\hline Lunes et $\mathrm{al}^{8}$ & & & & $x$ & & & $x$ & $x$ & & $x$ & $4 / 10$ \\
\hline Toledo et $a l^{9}$ & $x$ & & $x$ & $x$ & & & $x$ & $x$ & $x$ & $x$ & $7 / 10$ \\
\hline Segura et al $^{10}$ & $x$ & & $x$ & $x$ & & & $x$ & $x$ & $x$ & $x$ & $7 / 10$ \\
\hline Araújo et $a^{11}$ & $x$ & & $x$ & $x$ & & & $x$ & $x$ & $x$ & $x$ & $7 / 10$ \\
\hline Miotti de Moura et al ${ }^{12}$ & & & & & & & & & & & $0 / 10$ \\
\hline Fiorelli et $\mathrm{al}^{13}$ & & & & & & & & & & & $0 / 10$ \\
\hline
\end{tabular}

2. Alocação aleatória dos sujeitos; 3. Alocação oculta dos sujeitos; 4. Semelhança entre os grupos no início do estudo; 5 . Sujeitos cegos; 6 . Terapeutas cegos; 7. Avaliadores cegos; 8 . Mensuração de $85 \%$ dos resultados; 9 . Intenção do tratamento; 10. Comparação entre os grupos; 11 . Precisão e Variabilidade. 


\section{Discussão}

O objetivo principal do presente estudo foi averiguar quais seriam as possibilidades de tratamento descritas na literatura atual para o tratamento da escoliose e seus benefícios. Os estudos selecionados nesta revisão apresentaram maior amostra de participantes jovens e do sexo feminino, como já era esperado uma vez que a escoliose idiopática é uma condição com prevalência nesta população $7,8,9,10,11,12,13$. Wajchenberg et al. ${ }^{14}$, mostra em seu estudo que influências dos fatores genéticos e do meio ambiente sob a escoliose idiopática é pesquisada desde os tempos mais remotos e que ainda não se tem uma resposta definida. No entanto, sabe-se que a escoliose idiopática do adolescente é uma doença relacionada a fatores genéticos e que determinados grupos são mais suscetíveis, como indivíduos do sexo feminino ${ }^{14}$.

Um estudo realizado por Vieira et al. ${ }^{15}$ com objetivo de detectar sinais precoces da escoliose em crianças pré-escolares, encontrou respostas positivas no teste de Adams com gibosidade em forma de " $C$ ". E descreveu que pode se tratar de uma atitude escoliótica estando relacionada à fase de desenvolvimento musculoesquelético, não obrigatoriamente apresentando uma futura escoliose. Com achados em dominância literária a prevalência de $38,9 \%$ de gibosidade em meninas na faixa etária entre 8 e 15 anos de idade ${ }^{15}$.

Todos os estudos selecionados têm sua importância literária e na maioria houve uma melhora significativa no ângulo da escoliose ${ }^{9,10,13}$. A maioria dos estudos selecionados possuíram amostras reduzidas 7,8,9,10,12,13. Que juntamente com a demonstração incompleta de algumas informações influenciaram nos riscos de viés, após a análise foi obtido Score médio de 0,44 pontos pela escala de PEDro. É importante ressaltar que dois dos estudos se tratava de estudo de ca$\operatorname{sos}^{12,13}$. Os critérios do presente estudo em utilizar apenas artigos com tradução em português trouxe limitações para a pesquisa, porém esses fatores negativos não anulam os resultados encontrados.

Os estudos selecionados $7,8,9,10,11,12,13$ nos mostram que é comum o tratamento fisioterapêutico conservador com esta classe de pacientes e que seus resultados são favoráveis. Considerando a melhora dos benefícios já descritos e nenhum destes apresentou efeitos colaterais ou complicações para os participantes, inibindo assim a progressão da escoliose e reduzindo o risco de tratamento invasivo. Foram classificados como resultados positivos $81,08 \%$, da amostra geral dos estudos e 18,92\%, como sem alteração. Indicando que o tratamento fisioterapêutico para a escoliose é benéfico reforçando as evidências científicas e relevância para novos profissionais de saúde e outros usuários $7,8,9,10,11,12,13$.

Em um estudo de Viola et al. ${ }^{16}$ corroborando com os resultados encontrados nesta pesquisa sobre os benefícios da Fisioterapia, foi proposto um protocolo de reabilitação conservador para diferentes tipos de doenças da coluna através de sessões de Fisioterapia motora, cinesioterapia e meios físicos analgésicos, os quais apresentaram uma redução significativa no volume de cirurgias da coluna, diminuindo a exposição destes a procedimentos invasivos bem como a redução de custo cirúrgico final ${ }^{16}$.

A técnica do RPG reduziu a curva escoliótica nos dois estudos incluídos nesta revisão ${ }^{9,10}$. Uma revisão de literatura realizada por Teodori et al. ${ }^{17}$ foi encontrado 11 estudos envolvendo a aplicação da técnica de RPG para o tratamento de divergentes disfunções, através das principais posturas "Rã no chão e Rã no ar", apresentou resultados satisfatórios em geral quanto ao aumento de força e flexibilidade muscular, melhora da incontinência urinária, aumento de mobilidade cervical e alívio de dor crônica, melhora das funções respiratórias e melhora da capacidade funcional, comprovando que o RPG é uma boa técnica para prevenção e recuperação de disfunções musculoesqueléticas ${ }^{17}$, relacionando com o que foi observado.

No estudo realizado por Rossi et al. ${ }^{18}$ com mulheres jovens e saudáveis que apresentaram encurtamento muscular da cadeia anterior, utilizando apenas uma vez a técnica de RPG com a posição de "Rã no Chão" foi suficiente para melhorar a posição da cabeça e do ombro, de forma imediata após a aplicação da técnica. Esta postura tem como objetivo o alongamento dos músculos e fáscias da cadeia anterior do corpo, alinhando e reduzindo a tensão e sobrecarga nas articulações e músculos ${ }^{18}$. Este método corrobora com os resultados encontrados 9,10 por se mostrar eficaz na correção de alteração postural através do alongamento do tecido muscular promovendo o equilíbrio dos músculos responsáveis pela postura. 
Os exercícios do método pilates apresentaram benefícios na diminuição da dor crônica da coluna, meIhoria do padrão postural e flexibilidade ${ }^{11,12}$. Como foi visto, este método trabalha com exercícios musculares de baixo impacto, fortalecendo intensamente a musculatura estabilizadora da coluna. Conceição et al. ${ }^{19} \mathrm{em}$ seu estudo com relatos de casos sobre lombalgia crônica, constatou que o método pilates proporcionou melhora na estabilização da coluna e principalmente diminuição significativa da dor impactando na qualidade de vida diária das participantes ${ }^{19}$.

Após a investigação dos possíveis tratamento descritos na literatura para o tratamento da escoliose e seus benefícios, podemos afirmar que existem diferentes tipos de intervenções que podem ser empregadas no tratamento de desvios posturais. Isso não significa que há uma técnica melhor que outra, mas que elas podem ser complementares ou específicas para condições diferentes.

\section{Conclusão}

Diante dos estudos apresentados foi observado na literatura diversos métodos da Fisioterapia com benéficos para o tratamento da escoliose, destacando o RPG e o Pilates para a melhora da força, flexibilidade, dor e redução da curva escoliótica. Este resultado acontece através das diferentes técnicas e exercícios de fortalecimento muscular, alongamento e mobilidade da coluna vertebral. São necessários mais ensaios clínicos para reforçar as evidências sobre os métodos fisioterapêuticos na escoliose.

\section{Contribuições das autoras}

Medeiros SML e Freitas MGS participaram da concepção, delineamento, busca e análise estatística dos dados da pesquisa, interpretação dos resultados, redação do artigo científico. Camara GLG participou da orientação da pesquisa.

\section{Conflitos de interesses}

Nenhum conflito financeiro, legal ou político envolvendo terceiros (governo, empresas e fundações privadas, etc.) foi declarado para nenhum aspecto do trabalho submetido (incluindo, mas não se limitando a subvenções e financiamentos, participação em conselho consultivo, desenho de estudo, preparação de manuscrito, análise estatística, etc.).

\section{Referências}

1. Floyd RT. Manual de cinesiologia estrutural. 16.ed. Barueri, SP: Manole; 2011.

2. Mercante JIS, Soler FS, Myamoto CA. Recursos fisioterapêuticos para o tratamento da escoliose. Rev Conexão Eletrônica. 2019;16(1):1-9.

3. Petrini AC, Vaceslau AC, Oliveira LG, Colombo SJM. Fisioterapia como método de tratamento conservador na escoliose: uma revisão. Revista Científica FAEMA. 2015;6(2):17-35, 2015. doi: 10.31072/rcf.v6i2.308

4. Rocha, LF, Vitorino N, Parente D. Escoliose e suas bases genéticas. Revista saúde em foco. 2019;1(1):82-92.

5. Sociedade de pesquisa em escoliose. Dúvidas comuns sobre Escoliose. Scoliosis Research Society. [Internet]. 2019. [acesso em 2019 set. 10] Disponível em: <https://www.srs.org/portuguese/ patient_and_family/scoliosis/index.htm>

6. Alves AL, Sabino GS, Gomes RBO, Felício DC. Tratamento Fisioterápico na Escoliose. Pós em Revista. [Internet]. 2013. [acesso em 2020 set. 10]. Disponível em: http://blog.newtonpaiva. br/pos/wp-content/uploads/2013/04/PDF-E6-FISIOT36.pdf

7. Monte-Raso VV, Ferreira PA, Carvalho MS, Rodrigues JG, Marthins CC, lunes DH. Efeito da técnica isostretching no equilíbrio postural. Fisioterapia e Pesquisa. 2009;16(2):137-142. doi: $\underline{10.1590 / S 1809-29502009000200008}$

8. Iunes DH, Cecílio MBB, Dozza MA, Almeida PR. Análise quantitativa do tratamento da escoliose idiopática com o método klapp por meio da biofotogrametria computadorizada. Brazilian Journal of Physical Therapy. 2010;14(2):133-140. doi: 10.1590/ \$1413-35552010005000009

9. Toledo PCV, Melo DB, Araújo ME, Daoud R, Dantas EHM. Efeitos da Reeducação Postural Global em escolares com escoliose. Fisioter Pesq. 2011;18(4):329-334. doi: 10.1590/S180929502011000400006

10. Segura DCA, Nascimento FC, Guilherme JH, Sotoriva P. Efeitos da reeducação postural global aplicada em adolescentes com escoliose idiopática não estrutural. Ciências saúde UNIPAR. 2013;17(3):153-157.

11. Araújo MEA, Silva EB, Vieira PC, Cader SA, Mello DB, Dantas EHM. Redução da dor crônica associada à escoliose não estrutural, em universitárias submetidas ao método Pilates. Revista de Educação Física. 2010;16(4):958-966. doi: 10.5016/1980-6574.2010v16n4p958

12. Moura PM, Silva ML, Teixeira LP, Yamanda EF, Lara S. Efeito do método Pilates sobre a escoliose idiopática:estudo de caso. Sci Med. 2014;24(4):319-398. 
13. Fiorelli A, Arca EA, Fiorelli CM, Vitta A, Weckwerth PH, Strandman MTM et al. Redução da escoliose idiopática juvenil pós-intervenção cinesioterapeutica: relato de caso. Salusvita. 2014;33(3):355-363.

14. Wajchenberg M, Martins DE, Puertas EB. Aspectos genéticos da escoliose idiopática do adolescente. Coluna/Columna. 2012;11(3):234-236. doi: 10.1590/S1808-18512012000300010

15. Vieira DBALP, Beresoski CM, Camargo MZ, Fernandes KBP, Siqueira CPCM, Fujisawa DS. Sinais precoces de escoliose em crianças pré-escolares. Fisioterapia e pesquisa. 2015;22(1):69-75. doi: 10.590/1809-2950/13269222012015

16. Viola DCM, Lenza M, Almeida SLF, Santos OFP, Cendoroglo Neto $M$, Lottenberg CL et al. Redução do custo em cirurgia de coluna em um centro especializado de tratamento. Einstein. 2013;11(1):102-7. doi: 10.1590/S167945082013000100018

17. Teodori RM, Negri JR, Cruz MC, Marques AP. Reeducação Postural Global: uma revisão da literatura. Brazilian Journal of Physical Therapy. 2011;5(3):185-189. doi: 10.1590/S141335552011000300003

18. Rossi LP, Brandalize M, Gomes ARS. Efeito agudo da técnica de reeducação postural global na postura de mulheres com encurtamento da cadeia muscular anterior. Fisioter Mov. 2011;24(2):255-263. doi: 10.1590/S0103-51502011000200007

19. Conceição JS, Mergener CR. Eficácia do método Pilates no solo em pacientes com lombalgia crônica: relato de casos. Revista Dor. 2012;13(4):385-388. doi: 10.1590/S1806-00132012000400015

20. Shiwa SR, Costa LOP, Moser ADL, Aguiar IC, Oliveira LVF. PEDro: a base de dados de evidências em fisioterapia. Fisioter Mov. 2011;24(3):523-533. doi: 10.1590/50103-51502011000300017

21. Galvão TF, Pansani TSA, Harrad D. Principais itens para relatar Revisões sistemáticas e Meta-análises: A recomendação PRISMA. Epidemiol Serv Saúde. 2015;24(2), doi: 10.5123/S167949742015000200017 